\title{
Depression in a Case of Moyamoya Disease: A Case Report
}

\author{
Anuja Bendre ${ }^{1}$, Sagar Karia ${ }^{2}$, Nilesh Shah ${ }^{3}$, Avinash Desousa ${ }^{4}$
}

\begin{abstract}
Moyamoya disease is a rare disorder where there is a stenosis of the internal carotid arteries and its proximal branches. The disorder has variable presentations with neurological, cognitive and depressive features. The disorder may also be seen secondary to peri-infectious and neurological sequel. We present herewith a case of a 14-year-old girl with tuberculous meningitis and ventriculoperitoneal (VP) shunting having depressive features that was diagnosed on evaluation to have moyamoya disorder.
\end{abstract}

Keywords: Depression, Moyamoya, Tuberculosis, Tuberculous meningitis.

Indian Journal of Private Psychiatry (2019): 10.5005/jp-journals-10067-0039

\section{INTRODUCTION}

Moyamoya is a cardiovascular disease that predisposes patients to a stroke in association with progressive stenosis of intracranial internal carotid arteries and their proximal branches. ${ }^{1}$ The pathophysiology is reduced blood flow due to reduced anterior circulation of the brain which leads to a compensatory development of collateral vasculature by small vessels near the apex of the carotid, on the cortical surface, leptomeninges, and branches of the external carotid artery supplying the dura and the base of the skull. ${ }^{2}$ The disorder was first described as "hypoplasia of the bilateral internal carotid arteries", where there is an appearance of abnormally dilated collateral vessels on angiography that looks like "something hazy, like a puff of cigarette smoke", which is in Japanese referred to "moyamoya". ${ }^{3}$ The epidemiology of moyamoya reveals that the disorder is common in children who are approximately 5 years of age and adults in their mid-40s and the disorder is twice as common in female patients as male patients. ${ }^{4,5}$ Sometimes the sole presentation of moyamoya may be headache and giddiness, where headache is the sole symptom in around $25 \%$ of cases. ${ }^{6}$ We present herewith a case that was referred for psychiatric evaluation and was diagnosed as moyamoya disease.

\section{Case Description}

A 14-year-old Gujarati speaking young girl residing in Mumbai, who was the 3rd of five siblings. She was born of a non consanguineous marriage and had dropped out of school. She had for the last 4 years, a history of tubercular meningitis twice, once at three years of age and at 11 years of age. Ventriculo-peritoneal (VP) shunting had to be done both times. The patient also had a history of an episode of seizure prior to the first VP shunting surgery followed by another seizure episode at 11 years of age following which, she was put on anti-epileptics. She was born of a full-term vaginal delivery and had an uneventful perinatal period. All her developmental milestones were attained as per age. She developed tuberculous meningitis at the age of 3 years. She was under treatment with antituberculous medication for 9 months at that time and then joined school at 5 years of age. The parents noticed that she was unable to cope with her studies and fared poorly in academics. She had a history suggestive of being bullied in her class and there were no behavioral disturbances reported by the parents.

\author{
${ }^{1-4}$ Department of Psychiatry, Lokmanya Tilak Municipal General \\ Hospital and Lokmanya Tilak Medical College, Mumbai, Maharashtra, \\ India \\ Corresponding Author: Avinash Desousa, Department of Psychiatry, \\ Lokmanya Tilak Municipal General Hospital and Lokmanya Tilak \\ Medical College, Mumbai, Maharashtra, India, Phone: +91 9820696828, \\ e-mail: avinashdes888@gmail.com \\ How to cite this article: Bendre A, Karia S, Shah N, et al. Depression \\ in a Case of Moyamoya Disease: A Case Report. Ind J Priv Psychiatry \\ 2019;13(2):85-86. \\ Source of support: Nil \\ Conflict of interest: None
}

At the age of 12 years, she had to undergo VP shunting again. After this the FM have noticed a change in her behavior in the form of occasional anger outbursts, crying spells, easy irritability and episodes of restlessness where she would repeatedly try to run away from home, throw things impulsively and not recall the episode. The parents were unable to exactly discern the time of onset of these episodes of restlessness and impulsivity.

The child also reported giddiness and headache which was dull aching and holocranial. On probing she reported that she would feel sad for most part of the day and feel uninterested in life and living. She would also feel like ending her life because she suffered from tuberculosis and all the associated problems and felt that her parents were being burdened by her. She also needed assistance in her routine activities which upset her. There was no history suggestive of psychosis, mania or other psychiatric disorders. She had reduced sleep and appetite. She had not yet attained menarche.

There was no family history suggestive of psychiatric illness. On clinical evaluation, she was short at $134 \mathrm{~cm}$, she had strabismus and a dragging gait. On central nervous system examination, she had normal muscle tone in all 4 limbs and her power was $4 / 5$ in all limbs with reflexes and other part of the examination being within normal limits.

On mental status examination, she was conscious, cooperative and oriented in time, place and person. She maintained eye to eye contact well. Rapport was easily established and her attention was arousable and sustained. She conveyed her mood to be sad and affect was congruent to mood. Speech was normal and thought processes had depressive themes. No overt thought abnormality was noted. She conveyed active suicidal ideation.

(c) The Author(s). 2019 Open Access This article is distributed under the terms of the Creative Commons Attribution 4.0 International License (https://creativecommons. org/licenses/by-nc/4.0/), which permits unrestricted use, distribution, and non-commercial reproduction in any medium, provided you give appropriate credit to the original author(s) and the source, provide a link to the Creative Commons license, and indicate if changes were made. The Creative Commons Public Domain Dedication waiver (http://creativecommons.org/publicdomain/zero/1.0/) applies to the data made available in this article, unless otherwise stated. 
She denied any hallucinations. Her concepts and judgements were intact and insight was assessed to grade III out of V. A neurology opinion was sought and they advised a fresh neuroimaging study in the form of magnetic resonance imaging. The imaging study suggested features of secondary moyamoya disease with a nodular conglomerate lesion.

She was started on escitalopram $10 \mathrm{mg}$ for her depressive symptoms and the neurologist suggested that we start aspirin (75 mg), flunarizine (5 mg), methyl cobalamin and calcium. The patient claimed $80 \%$ improvement within 3 weeks of treatment. We had a clinical discussion about combining aspirin and escitalopram but keeping in mind the improvement the patient had shown decided to continue with the same under close clinical observation.

\section{Discussion}

Moyamoya disease is rare diagnosis and may be missed clinically by primary care physicians. It is important that it be kept in mind as a differential diagnosis when treating complex neurological cases where it may be diagnosed as a secondary phenomenon. ${ }^{7}$ In a study of 36 patients with the disorder, 11 reported cognitive impairment and 5 reported depression. ${ }^{8}$ Depression was reported in our case as well. Thus, sometimes cognitive impairment and depressive features may be the presenting symptoms in a case of moyamoya disease. Psychiatrists in clinical practice must keep this differential diagnosis in mind when handling cases of depression that may ensue following neurological insults as seen in our case.
Consultation liaison exchanges between neurology and psychiatry as in our cases shall help ascertain the diagnosis and ensure quick treatment enabling faster recovery to the patient.

\section{References}

1. Scott RM, Smith ER. Moyamoya disease and moyamoya syndrome. New Engl J Med 2009;360(12):1226-1237. DOI: 10.1056/ NEJMra0804622.

2. Czartoski T, Hallam D, Lacy JM, et al. Postinfectious vasculopathy with evolution to moyamoya syndrome. J Neurol Neurosurg Psychiatry 2005;76(2):256-259. DOI: 10.1136/jnnp.2004.041046.

3. Currie S, Raghavan A, Batty R, et al. Childhood moyamoya disease and moyamoya syndrome: a pictorial review. Pediatr Neurol 2011;44(6):401-413. DOI: 10.1016/j.pediatrneurol.2011.02.007.

4. Phi JH, Wang KC, Lee JY, et al. Moyamoya syndrome: a window of moyamoya disease. J Korean Neurosurg Soc 2015;57(6):408-414. DOI: 10.3340/jkns.2015.57.6.408.

5. Herve $D$, Touraine $P$, Verloes $A$, et al. A hereditary moyamoya syndrome with multisystemic manifestations. Neurology 2010;75(3):259-264. DOI: 10.1212/WNL.0b013e3181e8ee3f.

6. Takanashi JI. Moyamoya disease in children. Brain Dev 2011;33(3):229234. DOI: 10.1016/j.braindev.2010.09.003.

7. Kuroda S, Houkin K. Moyamoya disease: current concepts and future perspectives. Lancet Neurol 2008;7(11):1056-1066. DOI: 10.1016/ S1474-4422(08)70240-0.

8. Karzmark P, Zeifert PD, Tan S, et al. Effect of moyamoya disease on neuropsychological functioning in adults. Neurosurgery 2008;62(5):1048-1052. DOI: 10.1227/01.neu.0000325866.29634.4c. 